\title{
Comparison of Metaphorical Expressions of the Heart between Chinese and English
}

\author{
Gong Cheng ${ }^{1}$ \\ ${ }^{1}$ Center for Linguistics and Applied Linguistics, Guangdong University of Foreign Studies, Guangzhou, China \\ Correspondence: Gong Cheng, Center for Linguistics and Applied Linguistics, Guangdong University of Foreign \\ Studies, Guangzhou, China.
}

Received: January 25, 2021

Accepted: February 8, 2021

Online Published: February 18, 2021

doi: 10.5539/elt.v14n3p25

URL: https://doi.org/10.5539/elt.v14n3p25

\begin{abstract}
This study intends to provide a semantic analysis of metaphorical expressions containing the body-part term "heart" in Chinese and English. The discussion of these expressions revolves around four perceived roles of the heart. It is suggested that the metaphorical consequences have a bodily or psychological basis on our hearts. The comparison between Chinese and English shows that there exist some similarities and differences, which can be accounted for both by the commonality of bodily experiences unique to human beings and by the discrepancy of cultural modes from different countries. Finally, a revised model depicting the relationship between body, language, culture, and cognitive ability has been proposed.
\end{abstract}

Keywords: metaphorical expressions, heart expressions, comparison, Chinese, English

\section{Introduction}

Lakoff and Johnson (1980) claim that metaphor is pervasive in everyday life, not just in language but in thought and action. If it is true, then what we experience every day has something to do with metaphor. By the same token, body parts which function as the vehicle for human being's performance should be the bases for metaphorical expressions. Though the analysis of the critical roles that body parts play in our daily communication can date back to a long time ago, it is not until the occurring of the cognitive linguistics that special attention has been paid to bodily basis expressions. In the field of cognitive linguistics, linguists such as Johnson (1987) and Lakoff (1987) have contributed a lot to the establishment of the links between human body and meaning. Recently, research interest has extended to semantic studies of the links between human facial expressions and emotions across languages and cultures (Wierzbicka, 1993, 1999, 2000; Ning Yu, 2000, 2001, 2002). These studies have accumulated evidence and deepened our understanding of the relationship between body parts and meaning. However, further exploration is needed out of the following two concerns. First, previous research mainly focused on the external body parts, which are just one aspect of our body. Thus, it is of great necessity to take into consideration the internal body parts for the purpose of getting a whole picture of the relation between body parts and human emotions. Second, a comparative perspective should be adopted to enrich cross-sectional studies.

The body-part term "heart" in Chinese will be analyzed within the scope of internal body parts explicated in section 2. In section 3, data will be provided to explain how the heart as an internal body part is involved in conventionalized Chinese expressions in great length. What follows next is the analysis of English metaphorical expressions of the heart as well as the comparison between Chinese and English. In the final section a brief conclusion will be made.

\section{Literature Review}

Though expressions with body-part terms are common in our daily life, there is a lack of systematic analysis in this regard. To my knowledge, only one research conducted by Ning Yu (2002) has made a thorough analysis. According to Ning Yu (2002), body-part terms offer a perspective through which we can delve into the metaphorical extensions characterized with human bodily experience. In this study, body-part terms have been divided into two classes: those denoting external body parts and those denoting internal body parts or organs. Wierzbicka (1999) claimed that there was a difference between external and internal body-part terms with regard to metaphorical extensions. While the external body-part terms are initially metonymic, the internal body-part 
terms are believed to evoke bodily images which are essentially metaphorical.

As one of the most important metaphorical extensions, emotion expressions have a close link with internal body-part terms. Against this backdrop, Ning Yu (2002) made a detailed analysis of the emotions linked with the seven internal body parts including the spleen, the liver, the intestines, the stomach, the gall bladder, the lungs and the heart. Besides, images evoked by these internal body parts in Chinese have also been explored. For example, "spleen" is linked with the emotion of ANGER and the image of GASEOUS ENERGY EXPANDING. As one of the seven internal body parts, "heart" proves to be the most frequently used body-part term with various kinds of images embodied. This is because "heart" has been conceptualized as "commander" of all mental or psychological activities (Ning Yu, 2002). Thus, the semantic analysis of "heart" related expressions will facilitate our understanding of the metaphorical extensions about internal body-part terms.

Despite the pioneering work conducted by Ning Yu (2002), the shortcomings lie in that he just listed the various emotions conveyed by the "heart" related expressions in Chinese without considering the subtle differences between "heart" and "mind" which share the same Chinese character "xin". However, in other languages, for example English, "heart" and "mind" are two different words though they share the same Chinese equivalent "xin". Therefore, a comparison concerning "heart" related expressions between Chinese and English should be made to find the subtle differences on metaphorical extensions driven by human cognitive ability.

\section{Metaphorical Expressions of the Heart in Chinese}

Following the definition of "heart" by Modern Chinese Dictionary (the 7th edition), three different senses have been presented: (1) the organ in one's chest (2) thought or ideas (3) the central position. These three senses will serve as the bases for metaphorical extensions of "heart" related expressions in Chinese. At the outset, the criterion for the selection of metaphorical expressions of the heart in Chinese will be clarified. According to Kovecses (p4, 1986), although most expressions of a language can be regarded as conventional (except, maybe, sound-imitating words), not all conventional expressions are also conventionalized, that is, worn-out, clichéd, etc. Therefore, the expressions quoted in this study will be confined to conventionalized usage, namely, compounds or idioms.

\subsection{Heart as Sign of Emotion}

It seems that the relationship between body and emotion, especially the role of body in emotion and the impact of emotion on body, has aroused researchers' great interest in the past decades. For example, the research conducted by Ning Yu $(2000,2001,2002)$ has explored how our external body parts like "face", "hand", "finger" and "palm" interact with our emotion and figured out the way they are expressed metaphorically in Chinese. The results of his research corroborate the assumption that there is a strong correlation between body parts (both external and internal) and emotion language. Besides, the frequency of these metaphorical expressions involving body-part terms in Chinese varies greatly with different parts of the body taking up different proportions in usage. Among those internal body parts, "heart" stands out in emotion expression. In the following examples, "heart" is used to combine with other parts of body like "lungs" and "gallbladder" as well as words describing HEAT like "fire" and "coldness".

(1) a. xin-hua-nu-fang (heart-flower wildly-bloom) 'become extremely happy; burst with joy'

b. xin-kuang-shen-yi (heart-vast spirit-joyful) 'relaxed and joyful'

c. xin-zui (heart-drunk) 'be overcome with admiration'

d. si-xin-lie-fei (tear-heart split-lungs) 'be extremely grieved'

e. xin-ji-huo-liao (heart-anxious fire-flaming) 'burning with impatience; in a nervous state'

f. xin-huang-yi-luan (heart-flustered mind-puzzled) 'be alarmed and nervous; mentally confused'

g. xin-hui-yi-leng (heart-gray will-cold) 'be downhearted; be dispirited'

h. xin-han (heart-frigid) 'be bitterly disappointed'

i. xin-jing-dan-zhan (heart-shocked gallbladder-trembled) 'be panic-stricken; tremble with fear'

j. xin-xue-lai-chao (heart-blood coming-tiding) 'be seized with a sudden impulse'

k. xin-chao-peng-pai (heart-tide surging) 'be full of an excitement; feel an upsurge of emotion'

1. xin-ping-qi-he (heart-calm breath-smooth) 'be in a calm mood; become gentle with'

m. kuan-xin (broad-heart) 'be at ease; feel relieved'

In Chinese, it is a widely accepted metaphor that HEART IS THE SEAT OR CONTAINER FOR EMOTION 
(Ning Yu, 2002). Thus, the expressions shown above best illustrate how this metaphor works. In (1a) and (1b), the "heart" related expressions are used to describe HAPPINESS. Those who are happy may have "flowers blooming" inside or feel spacious inside. In (1c) another state of emotion, namely, ADMIRATION, is expressed in terms of heart. Here the word "drunk" shows that someone is deeply attracted by somebody or something else as a result of the 'alcohol' (the large amounts of emotion) in the 'container' (the heart). If we feel painful, then our heart and other internal body parts like lungs will be affected. Therefore, in (1d) the idiom shows vividly what grief one would suffer with the action of tearing one's heart and splitting one's lungs. When "heart" is combined with the word "fire", it gives an account of an anxious emotion. In (1e) this ANXIETY is obtained with the heat given off by the fire (intense emotion) inside. In (1f), the ANXIETY usage can also be found in another condition, where heart is influenced because of one's getting confused. On the contrary, "heart" can also be collocated with the word "coldness" illustrated in (1g) to convey the meaning of DEPRESSION. Besides, the degree of DEPRESSION becomes larger with the scale of coldness getting intensified. Hence, in (1h) the word "frigid" reflects that the temperature of the liquid (one's emotion) in the container (the heart) reaches 0 or below, meaning that a person is bitterly disappointed. Apart from "lungs", "heart" often co-occurs with another internal body part "gallbladder" to express FEAR. In (1i) FEAR can "shock the heart and make the gallbladder trembled". As the metaphor suggests, heart is the container for emotion. Therefore, emotion can be regarded as a kind of liquid or gas occupying the container. In both $(1 \mathrm{j})$ and $(1 \mathrm{k})$, the blood and tide are liquid inside. In this way, the rising of the 'tide' brings about EXCITEDNESS. In (11) the Chinese character qi represents a kind of gas. The concept 'gas' has a close connection with emotion, which is justified by the metaphor ANGER IS HOT GAS IN A CONTAINER in Chinese (Kovesces, 2000; Lakoff and Kovesces, 1987). In this example, one's mood is calm and the gas is not hot, thus gaining the meaning of CALM which is opposite to ANGER. In $(1 \mathrm{~m})$, which is similar to (1b), heart is seen as a container featured with breadth and spaciousness. In this case, it entails the meaning of RELIEF.

\subsection{Heart as Indicator of Personality}

The discussion of "heart" as sign of emotion has established links between one's inner world (the heart) and some psychological activities (the various mental reactions corresponding to different emotions). As is often the case, we are inclined to make a judgment about other people's characteristics or traits through their emotion. In other words, emotion is suggestive of personality. Based on these two types of relations including both "heart vs. emotion" and "emotion vs. personality", we seem to have reasons to believe that there should be some kind of link between "heart" and personality. In fact, we do find such expressions in Chinese. Let's take the following expressions as examples:

(2) a. xin-zhi-kou-kuai (heart-frank tongue-quick) 'be frank and outspoken; plain-spoken and straightforward'

b. xin-kou-ru-yi (heart-mouth consistent) 'be faithful to one's words; be frank and unreserved'

c. xin-ci-shou-ruan (heart-kind hand-soft) 'kindhearted and irresolute; be kind-hearted towards sb'

d. xin-hen-shou-la (heart-cruel hand-merciless) 'be extremely cruel and merciless; cold-hearted'

In (2a), "heart" keeps company with the external body-part term "mouth" through which the words are spoken. If the words sound credible and make others convinced, the speaker will leave an impression of honesty on the addressee. In English, we also have such expressions as "to be frank", "to be honest", "frankly speaking" etc. Since the heart is a hidden part and the addressee cannot see the speaker's heart directly, it is only through the words which make the addressee feel they are from the bottom heart of the speaker that the addressee can obtain such HONESTY impression. It is also true with the case in (2b). When the words are consistent with the speaker's heart, we tend to believe that the speaker possesses the personality of HONESTY. However, when "heart" is accompanied by another body-part term "hand", it often entails some negative or even evil personalities. In (2c), the "kind heart" combines with "soft hand" leading to the personality of IRRESOLUTION. Similarly, in (2d) the "kind heart" is in combination with "merciless hand" resulting in the personality of CRUELTY.

The following sentences quoted from the Center for Chinese Linguistics (CCL) corpus further demonstrate how we use "heart" related expressions to indicate our personalities.

$$
\begin{array}{crr}
\text { e. Wo xinkouruyi, } & \text { wo keyi wei-ci fashi. } \\
\text { I hear-mouth consistent } & \text { I } \quad \text { can for this swear. }
\end{array}
$$

I am faithful to my words, for which I can swear. 


$\begin{array}{ccccc}\text { f. Women } & \text { ju-buneng } & \text { dui } & \text { fanzui fenzi } & \text { xincishouruan. } \\ \text { We } & \text { never-can } & \text { treat } & \text { criminal members } & \text { heart-kind-hand-soft. }\end{array}$

We should never be kindhearted to the criminals.

\subsection{Heart as Pivot of Thought}

According to the definition of Chinese dictionary, "heart" can be referred to as thought or ideas. Maybe it is because of the "commander" role that heart plays in all kinds of psychological or mental activities that we treat heart as pivot of thought. The following idioms are illustrations of heart as thought or ideas.

(3) a. xin-bu-zai-yan (heart-absent) 'be absent-minded'

b. xin-yuan-yi-ma (hear-like ape mind-like horse) 'with one's mind running wild; one who can't settle down to his job'

c. xin-huai-po-ce (heart-breed evil strategy) 'have insidious aims in mind; have a sinister motive in mind'

d. xin-huai-gui-tai (heart-conceive ghost-embryo) 'conceive mischief; have sinister motives in mind'

e. xin-zhong-you-shu (heart have score) 'know one's own mind; have a pretty good idea of'

f. bie-chu-xin-cai (out of heart-fashion) 'be original in one's ideas; have an unconventional idea'

In (3a), it is a common phenomenon that one's heart is not present when getting involved in a certain mental activity. In that case, the person has been distracted by something else and thereby he or she is said to be absent-minded. The same is true of (3b), in which one's heart is agile as an ape and his mind is like a running horse. Both (3a) and (3b) show that "heart" related expressions convey the meaning of ABSENT-MINDEDNESS. If we compare heart to a womb, then strategies or plots are the embryos conceived in that womb. This "womb-embryo" metaphor makes it clear that the heart is the rich source of all kinds of strategies. In most cases, we are prone to choose strategies denoting mischief or sinister motives to combine with heart, which can be seen in (3c) and (3d). This negative meaning expressed by "heart" related expressions may be accounted for by the "hidden" feature of our heart. It is human nature that people might misbehave themselves especially when their conducts are out of supervision or cannot be monitored directly. For example, those who tell lies are a case in point. Lying itself is a mischief and the truth is hidden in the bottom of one's heart. In this situation, the meaning of MISCHIEF is delivered in the "heart" related expressions. There is always the chance that "heart" related expressions are employed to praise a good idea. In (3e) and (3f) a good or an unconventional idea is believed to originate from the heart. Thus, "heart" related expressions obtain the meaning of IDEA.

\subsection{Heart as Symbol of Treasure}

Just as face is the most distinctive external body part of a person, it is true that heart is the most vital internal body part for human beings. Without heart, none of physical activity is possible, let alone the psychological or mental activities. In this regard, heart outweighs other internal body parts and is seen as the most precious component. The following expressions, which are highly frequent in speech, are instances of heart as symbol of treasure.

(4) a. xin-gan (heart-liver) 'the person or thing that one loves most'

b. xin-jian (hear-tip) 'a sweetheart'

c. xin-ai (heart-love) 'prized possession; one's beloved'

In (4a), "heart" and "liver" which are two internal body parts of a person can be used to refer to the person or thing that someone loves most. In (4b) the tip of one's heart deserves special attention and be the apple of one's eye. In (4c) the love from the bottom of one's heart should be a precious possession or one's beloved. Based on these analyses, another meaning of VALUE can be added to "heart" related expressions.

\section{Metaphorical Expressions of the Heart in English and Comparison with Chinese}

The presentation of four perceived roles of heart in last section has demonstrated the bodily or psychological foundation that heart has laid for the metaphorical extensions in Chinese. Considering the commonality of bodily experiences of human beings, there is no doubt that we find similar metaphorical extensions in other languages, in particular English.

Before stepping further, we need to point out that the Chinese character "xin" has two different mappings in English: one refers to "heart" and the other denotes "mind". In this part, some of the "heart" and "mind" related 
expressions in English will be selected from Collins COBULD Advanced Learner's Dictionary (CCALD) from a comparative perspective. The comparison will lead us to the discovery of similarities and differences among "heart" or "mind" related expressions between English and Chinese. The following expressions in English will be ordered in accordance with their Chinese counterparts in section 3, namely, expressions linked with emotion come first and then with personality and thought and finally with treasure.

(5) a. win one's heart/lose one's heart to (EMOTION)

b. one's heart in one's mouth (EMOTION)

c. lose heart (EMOTION)

d. put/set one's mind at rest (EMOTION)

e. a load/weight off one's mind (EMOTION)

f. open one's heart; speak one's mind (PERSONALITY)

g. of two minds (PERSONALITY)

h. be absent-minded (THOUGHT)

i. with something in mind (THOUGHT)

j. close/near to one's heart (TREASURE)

As is shown above, expressions linked with emotion in English is disproportionally distributed for "heart" and "mind", of which "heart" takes greater proportion in expressing different emotions. In (5a) and (5c) the emotion of admiration and depression are expressed respectively. While in (5b) three different emotions are included: "anxiety", "excitedness" and "fear". Two "mind" related expressions are illustrated in (5d) and (5e) with the former linked with the emotion of calm and the latter of relief. When referring to the expressions linked with personality, "mind" seems to behave more actively than "heart" in this respect. A person who possesses the personality of irresolution is believed to be a person of two minds as reflected in (5g). However, (5f) suggests that an honest person is one who either speaks his mind or opens his heart. As to the expressions linked with thought, no "heart" related expressions can be found. Maybe this is due to the clear-cut between "heart" and "head" in English. Thought and ideas are regarded as products from one's head or mind instead of one's heart. Therefore, $(5 \mathrm{~h})$ is associated with the sense of absent-mindedness and (5i) the sense of ideas. The final example in (5j) shows the similarity between English and Chinese on "heart" related expressions in defining treasure. It is positioned in the center of our heart and is valued most by us.

Table 1. Comparison of "heart" related expressions between Chinese and English

\begin{tabular}{|c|c|c|c|}
\hline \multirow[b]{2}{*}{ Relevant senses in association with the body part of heart } & \multirow{2}{*}{$\begin{array}{l}\text { Chinese } \\
\text { "xin" }\end{array}$} & \multicolumn{2}{|l|}{ English } \\
\hline & & "heart" & "mind" \\
\hline 1.Happiness & + & & \\
\hline 2.Admiration & + & + & \\
\hline 3.Anxiety & + & + & \\
\hline 4.Depression & + & + & \\
\hline 5.Fear & + & + & \\
\hline 6.Excitedness & + & + & \\
\hline 7.Calm & + & & + \\
\hline 8.Relief & + & & + \\
\hline 9.Honesty & + & + & + \\
\hline 10.Irresolution & + & & + \\
\hline 11.Cruelty & + & & \\
\hline 12.Absent-mindedness & + & & + \\
\hline 13.Mischief & + & & \\
\hline 14.Idea & + & & + \\
\hline 15. Value & + & + & \\
\hline
\end{tabular}


Table 1 is a summary of the similarities and differences between Chinese and English in "heart" related expressions. As can be seen, the majority of the senses (except "happiness" and "cruelty") associated with the body part term "heart" in Chinese have equivalents in English, which further confirm our assumption that bodily and psychological basis of our heart contributes to the similarity of metaphorical extensions in different languages. Apart from these similarities, the differences are also obvious. While in Chinese an umbrella word "xin" is used to denote both "heart" and "mind", in English a clear distinction is made between the two. If we take a closer look at the usage of "heart" and "mind" in these English expressions, we can arrive at some tentative conclusions. Among these thirteen senses, seven are relevant to "heart" and six are linked with "mind". On the one hand, this result hints that "heart" and "mind" play almost equal important role in constructing Chinese equivalent expressions encompassing the body-part term "heart". On the other hand, "heart" is likely to be perceived as the sign of emotion or the symbol of treasure which is subjective and visible to some degree while "mind" tends to be seen as the indicator of personality or the pivot of thought which is objective and intangible in some way. Generally speaking, those metaphorical images, taken as a whole, reflect the Chinese and English way of constructing "heart" related expressions. However, this cross-sectional analysis is mainly linguistically based. In fact, further research needs to take into account the cultural factors which have material effect on the understanding of the expressions involving body-part term "heart" between Chinese and English.

\section{Conclusion}

In the final part, a tentative model designed for explaining the relationship between body, language, culture and cognitive ability in general is proposed. It is adapted from the "Triangle Model" put forward by Ning Yu (2002) and is shown by the diagram in Figure 1.

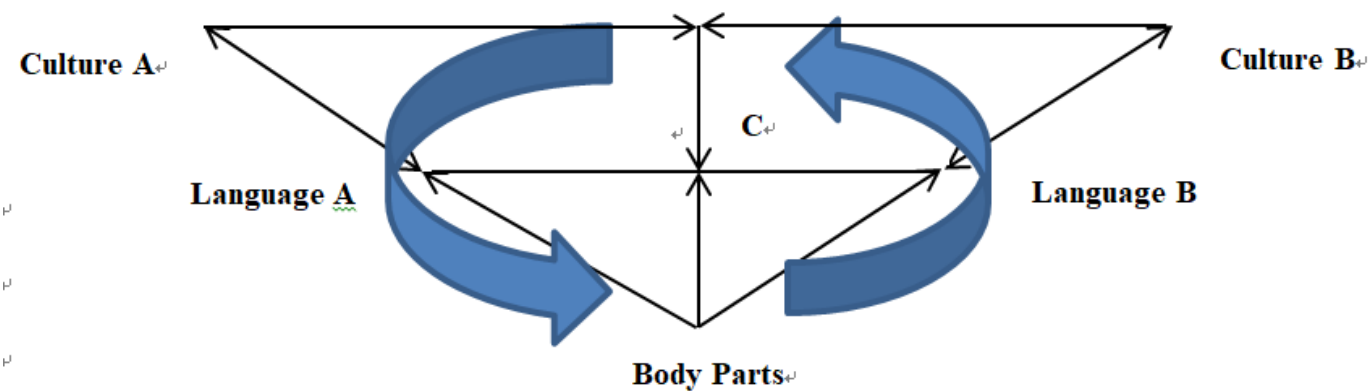

Figure 1. Revised Triangle Model (adapted from “Triangle Model” by Ning Yu 2002)

In this reversed triangle, body parts have been highlighted as the starting point on which our knowledge about the structure and functions of our body is based. Then our bodily experiences, both physical and psychological, will exert an impact on metaphorical expressions involving body-part terms in different languages. The line between language A and language B stands for the distance between two languages in conceptualizing body-part related expressions. These expressions of different languages will accordingly interact with different cultures and are finally shaped by their own cultural modes. The line between culture A and culture B represents the distance between two cultures in terms of their effect on metaphorical extensions of body-part related expressions. The distance at cultural level will affect the distance at linguistic level. However far culture A is away from culture B, they finally go back to body parts through language A and language B respectively. In fact, the whole process is a mirror of our cognitive ability, forming a dynamic system in which the three factors, namely, body parts, different languages and different cultures interact with one another. What's more, the central point " $\mathrm{C}$ " is the result of this interaction, which refers to the commonality existing between different languages and cultures.

\section{References}

Chinese Social Sciences Net. (2015). Center for Chinese Linguistics PKU: Modern Chinese Corpus. Retrieved from https://www.cssn.cn/yyx/yyxcyzy/201509/t20150922_2423138.shtml

Collins COBULD Advanced Learner's English Dictionary (8th ed.). [Z] (2014). Beijing: Foreign Language Teaching and Research Press.

Johnson, M. (1987). The Body in the Mind: The Bodily Basis of Meaning, Imagination and Reason. Chicago: University of Chicago Press. https://doi.org/10.7208/chicago/9780226177847.001.0001

Kovecses, Z. (1986). Metaphors of Anger, Pride and Love: A Lexical Approach to the Structure of Concepts. Amsterdam, Philadelphia: John Benjamins B.V. https://doi.org/10.1075/pb.vii.8 
Kovecses, Z. (2000). Metaphors and Emotions: Language, Culture, and Body in Human Feeling. Cambridge: Cambridge University Press.

Lakoff, G. (1987). Women, Fire, and Dangerous Things: What Categories Reveal about the Mind. Chicago: University of Chicago Press. https://doi.org/10.7208/chicago/9780226471013.001.0001

Lakoff, G., \& Johnson, M. (1980). Metaphors We Live By. Chicago: University of Chicago Press.

Lakoff, G., \& Kovecses, Z. (1987). The cognitive model of anger inherent in American English. In D. Holland \& N. Quinn (Eds.), Cultural Models in Language and Thought. Cambridge: Cambridge University Press. https://doi.org/10.1017/CBO9780511607660.009

Modern Chinese Dictionary. (7th ed.). [Z] (2012). Beijing: The Commercial Press.

Wierzbicka, A. (1993). Reading human faces: Emotion components and universal semantics. Pragmatics \& Cognition, 1(1), 1-23. https://doi.org/10.1075/pc.1.1.03wie

Wierzbicka, A. (1999). Emotions across Languages and Cultures: Diversity and Universals. Cambridge: Cambridge University Press. https://doi.org/10.1017/CBO9780511521256

Wierzbicka, A. (2000). The semantics of human facial expressions. Pragmatics \& Cognition, 8(1), 147-183. https://doi.org/10.1075/pc.8.1.08wie

$\mathrm{Yu}$, N. (2000). Figurative uses of finger and palm in Chinese and English. Metaphor and Symbol, 15(3), 159-175. https://doi.org/10.1207/S15327868MS1503_3

Yu, N. (2001). What does our face mean to us? Pragmatics \& Cognition, 9(1), 1-36. https://doi.org/10.1075/pc.9.1.02yu

Yu, N. (2002). Body and emotion: Body parts in Chinese expression of emotion. Pragmatics \& Cognition, 10(1/2), 341-367. https://doi.org/10.1075/pc.10.12.14yu

Yu, N. (2002). The bodily dimension of meaning in Chinese: What do we do and mean with 'hands'? In Eugene H. Casad \& Gary B. Palmer (Eds.), Cognitive Linguistics and Non-Indo-European Languages. Berlin: Mouton de Gruyter. https://doi.org/10.1515/9783110197150.8.337

\section{Copyrights}

Copyright for this article is retained by the author(s), with first publication rights granted to the journal.

This is an open-access article distributed under the terms and conditions of the Creative Commons Attribution license (http://creativecommons.org/licenses/by/4.0/). 